\title{
Natural Genomic Design in Sinorhizobium meliloti: Novel Genomic Architectures
}

\author{
Xianwu Guo, Margarita Flores, Patrick Mavingui, ${ }^{1}$ Sara Isabel Fuentes, \\ Georgina Hernández, Guillermo Dávila, and Rafael Palacios ${ }^{2}$
}

Centro de Investigación sobre Fijación de Nitrógeno, Universidad Nacional Autónoma de México. Apdo. postal 565-A, Cuernavaca, Morelos, México

\begin{abstract}
The complete nucleotide sequence of the genome of Sinorhizobium meliloti, the symbiont of alfalfa, was reported in 2001 by an international consortium of laboratories. The genome comprises a chromosome of 3.65 megabases (Mb) and two megaplasmids, pSymA and pSymB, of $1.35 \mathrm{Mb}$ and $1.68 \mathrm{Mb}$, respectively. Based on the nucleotide sequence of the whole genome, we designed a pathway of consecutive rearrangements leading to novel genomic architectures. In a first step we obtained derivative strains containing two replicons; in a second step we obtained a strain containing the genetic information in one single replicon of $6.68 \mathrm{MB}$. From this last architecture we isolated revertants containing two replicons, and from these we could return to the original architecture showing the three replicons. We found that the relative frequency of excision of cointegrated replicons is higher at the site used for the cointegration than at other sites. This might conciliate two apparently opposed facts: the highly dynamic state of genomic architecture in S. meliloti and the common observation that different isolates and derived cellular clones of $S$. meliloti usually present the architecture of one chromosome and two distinct megaplasmids. Different aspects that must be considered to obtain full advantage of the strategy of natural genomic design are discussed.
\end{abstract}

[Supplemental material is available online at www.genome.org.]

Bacterial genomes are dynamic structures. Homologous recombination between reiterated DNA sequences generates different types of genomic rearrangements (Anderson and Roth 1977; Petes and Hill 1988; Romero and Palacios 1997). The knowledge of the DNA sequence of a genome allows the precise location of the reiterated elements and thus the prediction of the different rearrangements that such a genome might generate. Bacterial subpopulations, pure or enriched for specific rearrangements, may be isolated by artificial selection procedures (Flores et al. 2000). Pathways of consecutive rearrangements leading to alternative genomic structures can be designed; derivative strains presenting the desired structures may be obtained by successive enrichment of bacterial subpopulations (Flores et al. 2000). In the present study, we applied the strategy of natural genomic design to obtain novel genomic architectures in Sinorhizobium meliloti, the symbiont of alfalfa.

Bacteria of the genus Rhizobium and related genera interact with the roots of leguminous plants, establishing nitrogen-fixing symbioses. The Rhizobium genome usually contains a large amount of reiterated DNA sequences (Flores et al. 1987; Freiberg et al. 1997; Kaneko et al. 2000) and presents genomic rearrangements at high frequency (Flores et al. 1988, 2000; Romero and Palacios 1997; Mavingui et al. 2002). We recently showed that genome architecture is a dynamic process in the broad host range Rhizobium sp NGR234 (Mavingui et al. 2002).

The genome of Sinorhizobium meliloti 1021 comprises three replicons: a chromosome of $3.65 \mathrm{Mb}$ and two megaplasmids pSymA and pSymB, of $1.35 \mathrm{Mb}$ and $1.68 \mathrm{Mb}$, respectively. The complete nucleotide sequence of the three replicons has been

\footnotetext{
'Present address: Écologie Microbienne, UMR CNRS 5557, Université Claude Bernard, Lyon 1, France. ${ }^{2}$ Corresponding author.

E-MAIL palacios@cifn.unam.mx; FAX (777) 311-4660.

Article and publication are at http://www.genome.org/cgi/doi/10.1101/ gr.1260903.
}

reported (Barnett et al. 2001; Capela et al. 2001; Finan et al. 2001; Galibert et al. 2001). The availability of the nucleotide sequence, together with the extensive genetic and biochemical knowledge accumulated in S. meliloti, make this organism an ideal model for genome dynamic studies. Based on the nucleotide sequence, we located the major reiterated elements and predicted the recombination events that might produce changes in the global architecture of the genome. Starting with the wild-type $S$. meliloti strain 1021, we obtained derived strains presenting different genomic architectures.

\section{RESULTS}

\section{Rationale for the Natural Genomic Design in S. meliloti}

The main goal of the genomic design was to obtain an S. meliloti derivative strain containing all the genetic information in a single replicon. The annotation of the nucleotide sequence of the three replicons of $S$. meliloti was analyzed, and the reiterated sequences shared by more than one replicon were localized. The potential rearrangements leading to cointegration between replicons were predicted. The actual occurrence of several cointegration events was identified in a culture of the wild-type strain $S$. meliloti 1021, following a PCR-based approach.

In the first step of the design, the wild-type culture was plated, and colonies presenting only two replicons, one being the result of cointegration, were selected. The artificial selection procedure used was the analysis of replicon profile using agarose gel electrophoresis. In the second step of the design, a culture of a derivative strain containing the chromosome and a replicon derived from the cointegration of pSymA and pSymB was plated, and the replicon profile of individual colonies was analyzed. A derivative strain containing only one replicon (a cointegrate of the chromosome and the two megaplasmids) was selected. From this strain, two additional steps led to the original three-replicon architecture. 
I
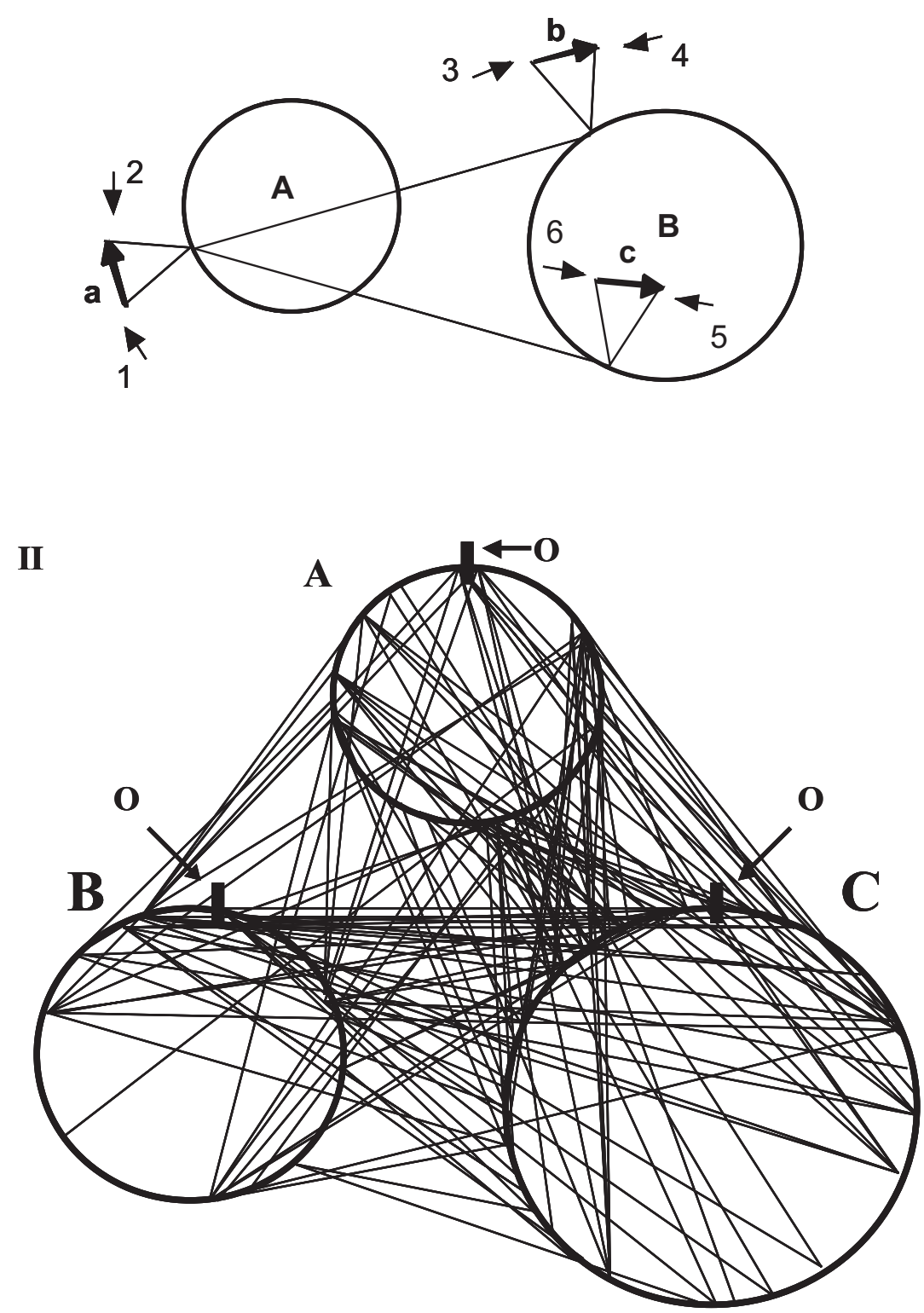

Figure 1 Diagram of the potential sites for replicon cointegration in the S. meliloti genome. (I) Schematic representation of the points of recombination between a repeated family containing 1 element $(a)$ in replicon A and two elements $(b, c)$ located in inverted orientation in replicon $B$. Each element is represented by a large arrow indicating the orientation; the small arrows indicate the primers bordering each element. Primers are indicated by numbers: 1 , primer at the $5^{\prime}$-end of element $a ; 2$, primer at the $3^{\prime}$-end of element $a ; 3$, primer at the $5^{\prime}$-end of element $b ; 4$, primer at the $3^{\prime}$-end of element $b ; 5$, primer at the $3^{\prime}$-end of element $c ; 6$, primer at the 5 -end of element $c$. The lines linking the two replicons indicate potential points for recombination leading to cointegration. To detect cointegration by the PCR method (see text), the following primers are used: for cointegration by $a$ and $b$ elements, primers 1 and 4 or 2 and 3; for cointegration by $a$ and c elements, primers 1 and 5 or 2 and 6. (II) The three replicons of the genome of S. meliloti 1021 are represented by circles: A, pSymA, B, pSymB, C, chromosome. The area is proportional to the length of each replicon. The origin of replication is represented by $\mathrm{O}$; the direction of the annotation is clockwise according to that reported (Galibert et al. 2001; http://sequence.toulouse.inra.fr/meliloti.html). Repeated sequences with more than $95 \%$ sequence similarity in a track of at least $1 \mathrm{~kb}$ and present in more than one replicon were localized. Each pair of repeated sequences that might recombine generating replicon cointegrations is represented by a line linking two replicons at the corresponding sites of the repeats.

\section{Localization of Reiterated Sequences Shared by More Than One Replicon}

Based on the annotation of the sequence of each of the three replicons (Barnett et al. 2001; Capela et al. 2001; Finan et al. 2001; http:// sequence.toulouse.inra.fr/meliloti.html), the reiterated elements presenting more than 95\% sequence similarity at the nucleotide level in a continuous track of at least $1 \mathrm{~kb}$ were localized. These elements included both insertion sequences (ISs) and specific genes or groups of genes. We found a total of 133 pairs of repeated elements that could lead to the cointegration of two replicons by homologous recombination. From these, 123 pairs corresponded to IS elements, and 10 pairs corresponded to duplicated genes. A schematic map of the potential sites to form cointegrates between two replicons is shown in Figure 1. A table listing the pairs of the repeated elements is presented as Supplementary Material (available online at www.genome.org).

\section{Identification of Cointegration Events}

We showed previously that rearrangements can be identified in a bacterial culture using a PCR-based strategy (Flores et al. 2000). Primers for PCR are synthesized in the proximity of the two reiterated sequences involved in a potential rearrangement. If an appropriate combination of primers from two replicons is used (see Fig.1I), the corresponding PCR product indicates the occurrence of the cointegration in some of the cells of the culture. Since cells with specific rearrangements are highly diluted in the culture, the amount of PCR product obtained is usually low, and a large number of reaction cycles must be used. Moreover, it is our experience that to detect some rearrangements, several alternative combinations of primers should be tested (Flores et al. 2000).

We prepared pairs of primers for each of the reiterated elements presenting the abovementioned characteristics of size and homology, that could lead to cointegration between two replicons in the $S$. meliloti genome. Following the PCR strategy, we analyzed the presence of all of the different potential cointegrates in the culture of wild-type $S$. meliloti 1021 . From 133 potential sites of cointegration between two replicons, we obtained positive PCR signals in 91 cases. From those that did not produce a positive signal, we randomly selected six cases and performed the PCR reaction using different combinations of alternative primers. In all the cases, we obtained positive reactions with some combinations of primers. In addition, we randomly selected some PCR products and sequenced both ends (data not shown), ascertaining that the sequence corresponded to the expected regions in each of the two replicons involved in the cointegration. The pairs of repeated sequences that produced positive PCR reactions and the position of the corresponding primers are indicated in the table presented 


\section{S. meliloti 1021}

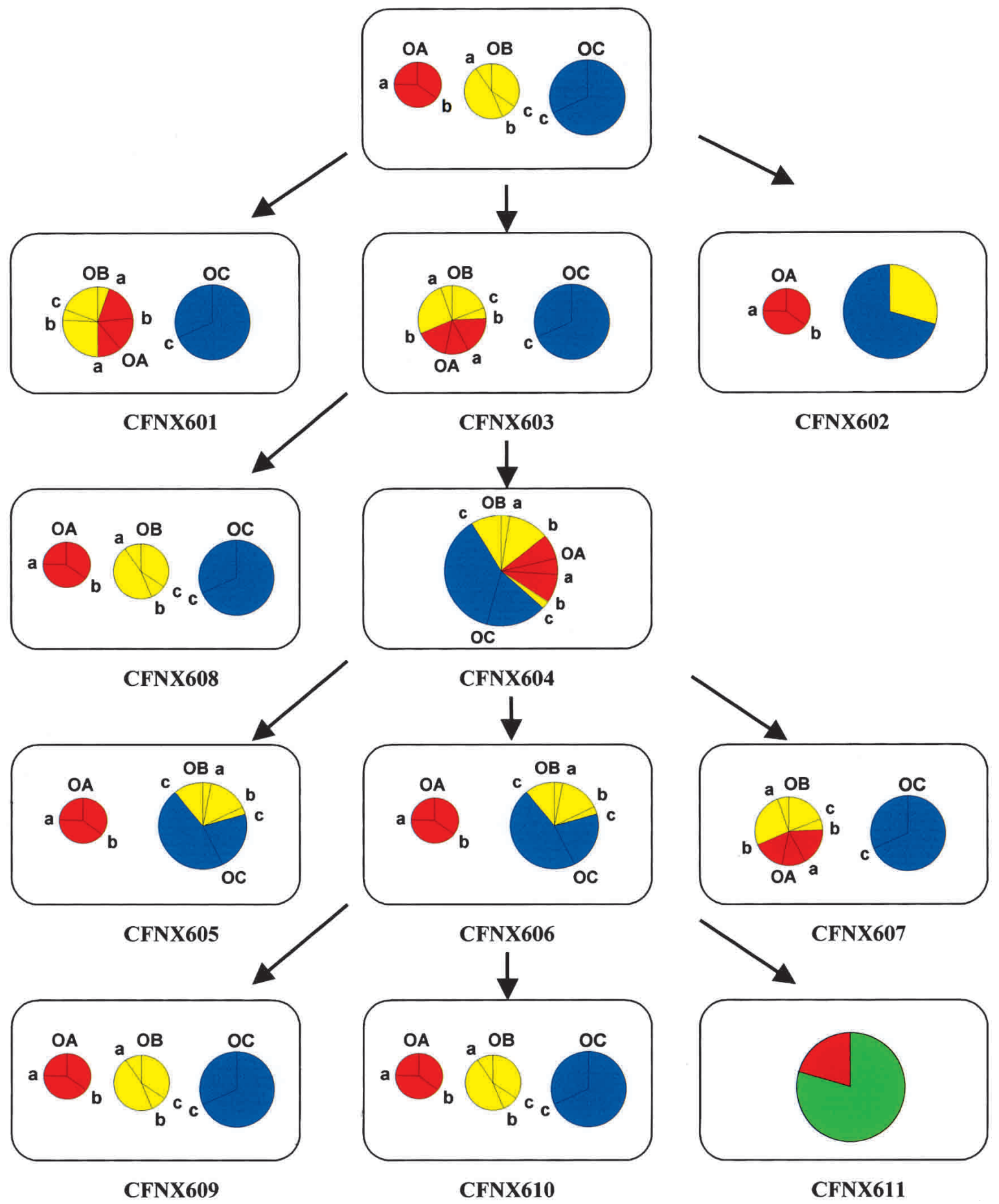

Figure 2 Diagram of derivative strains obtained in the genomic design applied to S. meliloti 1021. Original replicons are shown in color: red, pSymA; yellow, pSymB; blue, chromosome. Origins of replication are indicated as OA, OB, and OC for pSymA, pSymB, and chromosome, respectively. The sites of reiterated elements participating in cointegration are indicated: $a$, ISRm11; $b$, nodPQ genes; $c$, algl genes. The name of each derivative strain is indicated at the bottom of the corresponding diagram. The sites of cointegration of pSymB and the chromosome in strain CFNX602 and the site of cointegration of pSymA into the cointegrate formed by pSymB and the chromosome (represented in green) in strain CFNX611 are not known. 
as Supplementary Material. These data suggest that most of the potential sites are indeed used to produce cointegrations between replicons, and that cells containing such cointegrates are present in a culture of the wild-type $S$. meliloti 1021.

\section{Isolation of Novel Genomic Architectures}

As suggested above, a culture of the wild-type strain $S$. meliloti 1021 contains cells presenting different types of replicon cointegrates. The culture was plated in rich medium, and the replicon profile of 500 individual colonies was analyzed. Three colonies showed an altered architecture: strains CFNX601 and CFNX603 presented the chromosome and a novel replicon, product of the cointegration of the two megaplasmids; strain CFNX602 presented pSymA and a novel replicon, product of the cointegration between the chromosome and pSymB (Fig. 2). The site of cointegration of pSymA and pSymB in strain CFNX601 corresponded to ISRm11, whereas that in strain CFNX603 corresponded to the reiterated nodPQ genes.

Strain CFNX603 was selected to continue the genomic design. A culture of this strain was plated in rich medium, and the replicon profile of 500 individual colonies was analyzed. Two colonies presented genomic architecture different from that of CFNX603. Strain CFNX604 presented only one replicon: a novel structure of about $6.68 \mathrm{Mb}$, product of the cointegration of the three original replicons (Fig. 2). The sites of cointegrations were analyzed. As expected, the site of cointegration of pSymA and pSymB is the same as in strain CFNX603. The site of cointegration of the pSymA-pSymB replicon to the chromosome corresponds to the reiterated algI genes present in pSymB and in the chromosome. The other strain derived from CFNX603, CFNX608, presented the three original replicons. The site of excision of pSymA and pSymB was found to be the same used for the original cointegration: nodPQ genes. This strain is thus a revertant to the wild-type architecture.

Strains CFNX603 and CFNX604 were further characterized by pulsed-field gel electrophoresis and hybridization with probes specific for each of the three replicons (Fig. 3), ascertaining the structure of the corresponding cointegrates. Strain CFNX604 was used to study the influence of genomic architecture on growth and symbiotic properties (see below).

The stability of the architecture containing all the information in a single replicon, strain CFNX604, was studied under laboratory conditions. The strain was cultured in rich medium, with transfer of aliquots to fresh medium each day. After $30 \mathrm{~d}$, the culture was plated and the replicon profile of 500 individual colonies was analyzed. Only three colonies presented architec- tures different from that of CFNX604. The three colonies correspond to revertants to architectures containing two replicons. Strains CFNX605 and CFNX606 present pSymA and a cointegrate between pSymB and the chromosome; strain CFNX607 presents the chromosome and a cointegrate between pSymA and pSymB (Fig. 2). The excision between pSymA and pSymB in strains CFNX605 and CFNX606 and that from pSymB and the chromosome in strain CFNX607 were through the same sites of the original cointegrations.

A culture of strain CFNX606 was plated, and the replicon profile of 500 individual colonies was analyzed. Three strains showed architecture different from that of CFNX606. Two of them, strains CFNX609 and CFNX610, correspond to revertants to the original architecture. The excision site of pSymB and the chromosome was the same as that used for the original cointegration, the algI genes. In the other strain, CFNX611, pSymA was cointegrated to the replicon formed by pSymB and the chromosome, resulting in another strain containing only one replicon (Fig. 2). The site of cointegration of pSymA was not analyzed.

\section{Reversibility of Replicon Cointegration}

A cointegrate between two replicons contains different pairs of repeated sequences that might be used for excision. If a cointegrate is excised at the same site used for cointegration, it will generate revertants to the original replicons. Excision at other sites would generate novel complex replicons. During the experiments presented above, we obtained six excisions from complex replicons. It is interesting that in all cases the excisions were precisely at the original sites used for the cointegration. The data obtained in the experiment shown in Figure 4 and Table 1 also suggest that the preferential sites for replicon excision are those used for the initial cointegration. Strains CFNX601 and CFNX603 present a similar two-replicon-genome architecture: the chromosome and a pSymA-pSymB cointegrate. However, the site of cointegration is different in both strains: ISRm11 in CFNX601 and nodPQ genes in CFNX603. If the cointegrate is excised at ISRm11 in strain CFNX601 or at nodPQ genes in strain CFNX603, the original replicons, pSymA and pSymB, would be obtained. Conversely if the cointegrate is excised at nodPQ genes in CFNX601 or at ISRm11 in CFNX603, novel complex replicons would be obtained (see Fig. 4).

Cultures of both strains were analyzed for the presence of structures resulting from the excision of the pSymA-pSymB cointegrate at both sites, ISRm11 and nodPQ genes. The PCRbased strategy for identification of rearrangements (Flores et al. 2000; Mavingui et al. 2002) was used. The scheme presented in

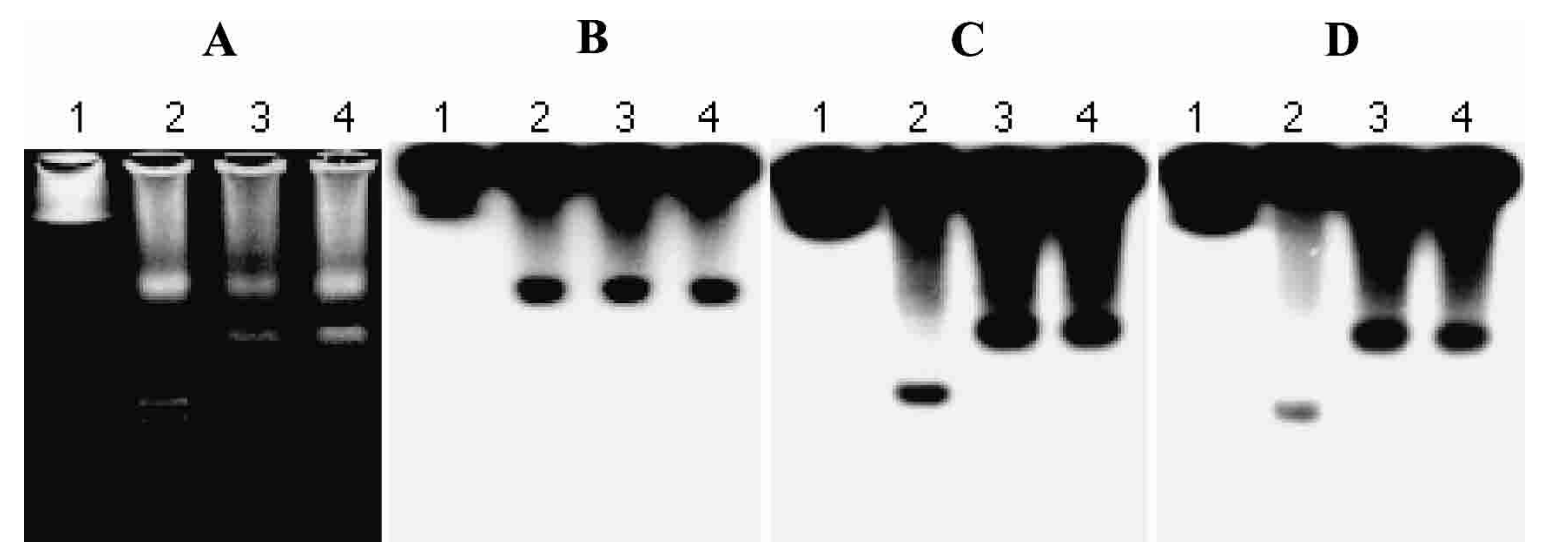

Figure 3 Replicon structure of S. meliloti 1021 and derivative strains. Pulsed-field gel electrophoresis of S. meliloti 1021 (lane 2), CFNX604 (lane 1), CFNX601 (lane 3), and CFNX603 (lane 4). (A) Agarose gel stained with ethidium bromide, (B) hybridization with rDNA probe, (C) hybridization with exo genes probe, and $(D)$ hybridization with nif genes probe. 


\section{S. meliloti 1021}
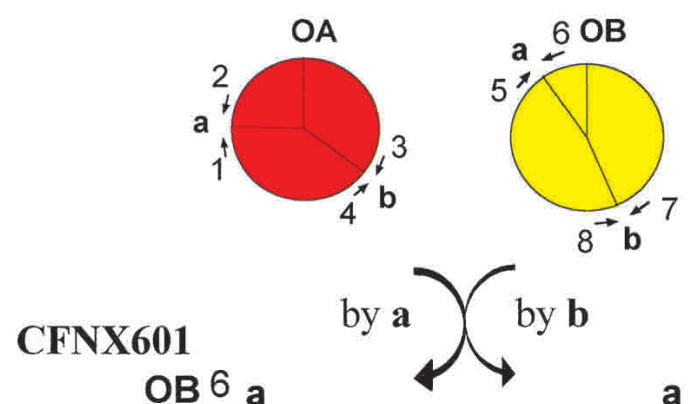

CFNX603 a 6 OB

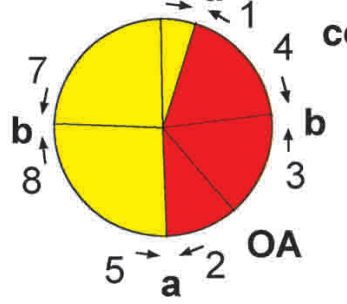

cointegration
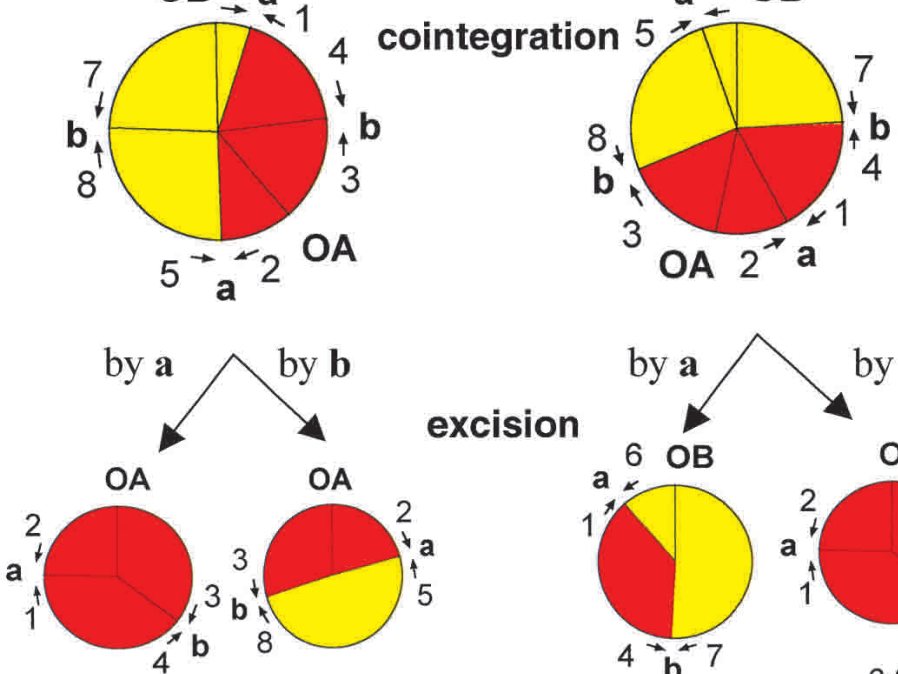

excision

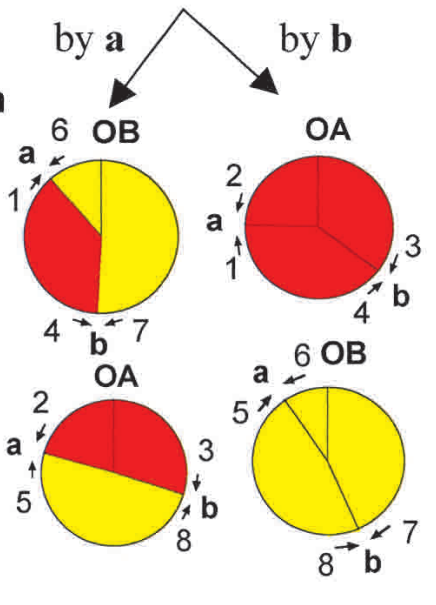

Figure 4 Diagram of cointegration and excision of replicons at alternative sites. The two megaplasmids of S. meliloti 1021 are represented in colors: red, pSymA; yellow, pSymB. The origins of replication are shown as $\mathrm{OA}$ for $\mathrm{pSymA}$ and $\mathrm{OB}$ for $\mathrm{pSymB}$. Alternative sites for cointegration and excision are indicated: a, ISRm11; b, nodPQ genes. Oligonucleotides bordering the reiterated elements participating in cointegration and excision events are indicated by arrows and numbers. The structures resulting from alternative sites of cointegration of pSymA and pSymB as well as those resulting from alternative sites of excision of the pSymApSymB cointegrates are shown.

Figure 4 shows the original structures and those expected as a result of cointegrations and excisions in the wild-type $S$. meliloti 1021, as well as in the derived strains CFNX601 and CFNX603. The locations of the PCR primers used in the experiment are indicated for the different genomic architectures. The appropriate combination of primers allowed the detection of the different structures. The relative amount of the corresponding PCR product indicated the relative concentration of each structure in the different cultures (Table 1). In both strains presenting a tworeplicon architecture, CFNX601 and CFNX603, the relative concentration of the structures derived from excision at the site of the original cointegration is higher than that of the structures derived from excision at the alternative site studied. This is in accordance with the observations presented in Figure 2, and suggests that once a cointegrate is formed, the probability of exci- sion at the site of the original cointegration is higher than at other sites.

\section{Influence of Genome Architecture on Growth and Symbiotic Properties}

The growth rate and symbiotic properties of wild-type strain S. meliloti 1021 were compared with those of a strain that presents the genetic information in a single $6.68-\mathrm{Mb}$ replicon (CFNX604; Fig. 5). To determine the growth in complete and minimal medium, three experiments with three replicate tubes each were performed. The three experiments gave similar results; one such experiment is presented in Figure $5 \mathrm{~A}, \mathrm{~B}$. In complete medium, the growth is exponential and there is no significant difference between the strains. In contrast, in minimal medium the growth is not exponential; in the three experiments performed, strain CFNX604 presented slightly but significantly lower growth compared to the wild-type strain.

In regard to the symbiotic properties, the number of nodules formed in growth pouches at different stages after inoculation was slightly higher in strain CFNX604 than in the wildtype strain; however, the total number of nodules formed and the foliage dry weight in pots were not significantly different.

\section{DISCUSSION}

To obtain full advantage of the strategy of natural genomic design, several aspects must be considered. The nucleotide sequence of the replicons that constitute the genome should be complete and closed. This is actually the case for the S. meliloti genome (Barnett et al. 2001; Capela et al. 2001; Finan et al. 2001). Draft sequencing without a complete assembly (Fraser et al. 2002) greatly limits the potentiality of the method and could give rise to misleading interpretations.

Because the length and degree of homology have strong effects on homologous recombination, best results are obtained when the design is based on large reiterated sequences with a high degree of sequence similarity. As shown in this study for replicon cointegration, repeated sequences larger than $1 \mathrm{~kb}$ and with more than 95\% sequence similarity are good targets for genome rearrangements leading to replicon cointegration and excision. Moreover, the sequences analyzed in this study can also recombine generating amplifications, deletions, and inversions when they are repeated in the same replicon (data not shown). Interesting designs might be made by combining the different types of rearrangements that can be generated by homologous recombination.

Rearrangements may also be generated by site-specific recombination mediated by specific enzymes. However, these events cannot be predicted by the nucleotide sequence alone and could be used for genomic design only when previous experimental knowledge is available.

At each step of the genomic design, it is important to identify the presence of the next rearrangement in the bacterial culture of the mother strain. The PCR-based approach (Flores et al. 2000) is ideal for this purpose, because it can detect structures 
Table 1. Quantification of Structures Resulting From Cointegration and Excision Events in the Cultures of Wild-Type S. meliloti 1021 and Its Derivative Strains

\begin{tabular}{|c|c|c|c|c|c|c|}
\hline Event & Site $^{a}$ & Primers $^{\mathrm{a}}$ & Control strain & Test strain & $\mathbf{n}^{\mathbf{b}}$ & $\mathbf{F}^{\mathrm{c}}$ \\
\hline \multirow[t]{4}{*}{ Cointegration of pSymA,pSymB } & $a$ & $1-6$ & CFNX601 & wild type & 12 & $2.4 \times 10^{-4}$ \\
\hline & a & $2-5$ & CFNX601 & wild type & 13 & $1.2 \times 10^{-4}$ \\
\hline & $\mathrm{b}$ & $3-8$ & CFNX603 & wild type & 12 & $2.4 \times 10^{-4}$ \\
\hline & $\mathrm{b}$ & $4-7$ & CFNX603 & wild type & 13 & $1.2 \times 10^{-4}$ \\
\hline \multirow[t]{4}{*}{ Excision from CFNX601 } & a & $1-2$ & wild type & CFNX601 & 10 & $1 \times 10^{-3}$ \\
\hline & $a$ & $5-6$ & wild type & CFNX601 & 10 & $1 \times 10^{-3}$ \\
\hline & $\mathrm{b}$ & $3-8$ & CFNX603 & CFNX601 & 12 & $2.4 \times 10^{-4}$ \\
\hline & $\mathrm{b}$ & $4-7$ & CFNX603 & CFNX601 & 13 & $1.2 \times 10^{-4}$ \\
\hline \multirow[t]{4}{*}{ Excision from CFNX603 } & $a$ & $1-6$ & CFNX601 & CFNX603 & 12 & $2.4 \times 10^{-4}$ \\
\hline & a & $2-5$ & CFNX601 & CFNX603 & 12 & $2.4 \times 10^{-4}$ \\
\hline & $\mathrm{b}$ & $3-4$ & wild type & CFNX603 & 10 & $1 \times 10^{-3}$ \\
\hline & $\mathrm{b}$ & $7-8$ & wild type & CFNX603 & 10 & $1 \times 10^{-3}$ \\
\hline
\end{tabular}

asite of event and position of the primers are shown schematically in Fig. 4. The sequence and location of the primers are given in Methods.

$b$ " $n$ " represents the difference in the number of cycles necessary to obtain the same amount of PCR product between the test and the control (see Methods). $n$ values are the mean of four independent measurements.

" $F$ " represents the relative concentration of rearranged structures in the culture (see Methods).

highly diluted in the population. Rearrangements produced by recombination of long and highly homologous reiterated sequences are usually generated at relative frequencies of $10^{-3}$ $10^{-6}$. This allows the inference that a bacterial culture should contain cells presenting each of the different rearrangements that the genome can generate, providing that they are not deleterious for growth in the particular conditions used. As shown in the present study, the PCR-based method revealed evidence, in the wild-type culture, of the presence of most of the predicted replicon-cointegration events. It is important to recall that to detect some rearrangements, different combinations of appropriate primers should be tested.

The relative frequency at which a particular rearrangement is present in a culture can be estimated by a quantitative PCR approach. Examples are shown in Table 1. Because different PCR primers result in different relative concentrations of PCR products, a quantitative approach is only valid if the appropriate control is used as a standard. In the experiment presented in Table 1, the amount of the corresponding PCR products obtained from the DNA of the wild-type strain was used as the control for the revertant excision events in strains CFNX601 and CFNX603. Conversely, the amount of PCR product corresponding to the joint point generated through recombination between ISRm11 using the DNA of strain CFNX601, and that corresponding to a recombination between nodPQ genes in strain CFNX603, were used as standards for the cointegration events in the wild type.

Once a particular rearrangement of the design is ascertained to be present in the mother strain, a subpopulation pure for such rearrangement must be isolated. It is important to recall that in the case of rearrangements that can revert, such as amplifications, inversions, and cointegrations, a population cannot be pure in a precise sense, because some cells would contain the corresponding revertant structures. A wild-type strain contains cells presenting different types of genomic rearrangements. To enrich a subpopulation for a specific rearrangement, without knowledge of its biological consequences, artificial selection procedures should be used. If the biological consequences of the desired rearrangement are known, selective pressure might be applied. In this study we used replicon profiles as the screening procedure, because we were not interested in the specific site of the desired cointegrations. When rearrangements at specific sites are desired, a PCR-based artificial selection can be used (Flores et al. 2000).

The main goal of the genomic design applied to $S$. meliloti in the present study was the isolation of a strain with all its genetic information in a single replicon, strain CFNX604. This strain presented similar growth in rich medium and nodulation capacity compared to the wild-type strain. These data suggest that genome architecture can be manipulated in $S$. meliloti without impairing physiological functions, at least under certain conditions in the laboratory. As presented here, although novel genome architectures generated by cointegration of different replicons have a tendency to revert, they can be maintained under laboratory conditions for relatively long periods of time. Furthermore, a strain with a particular architecture can be repurified from a culture by plating and analyzing individual colonies.

As shown in this study, genome architecture is a highly dynamic process in $S$. meliloti. On the other hand, different isolates and derived clones of $S$. meliloti usually present the architecture containing the chromosome and two distinct megaplasmids, pSymA and pSymB. Data presented in this study help to solve this apparent paradox. The strain containing only one replicon (CFNX604) showed a slower growth rate in minimal medium than the wild-type strain (Fig. 5). Strains with two replicon architectures presented higher growth than CFNX604 but lower than the wild type (not shown). In nature, strains are growing usually under limitation of nutrients; a slight difference in growth rate under such conditions would favor the presence of strains with the three-replicon architecture.

As shown here, $S$. meliloti replicons are continuously being cointegrated and excised. Once a cointegrate is formed, it might be excised through homologous recombination in different sites. This would generate novel complex replicons. Actually we detected structures corresponding to complex replicons, albeit at a very low relative frequency, in the data presented in Table 1 . If the frequency of excision events is similar for different sites, in the long term genetic information would be reshuffled among different replicons. However, the derived strains obtained in different steps of the design, and the data presented in Table 1, suggest that once a cointegrate is formed, the preferential site for excision is the same as that used for cointegration. This will tend to maintain the same replicons even under a highly dynamic state. The molecular basis for the preference of the excision site is not known at present.

The natural genomic design strategy was previously suggested using as a model the symbiotic plasmid of Rhizobium sp. NGR234 (Flores et al. 2000; Mavingui et al. 2002). The availability of the nucleotide sequence (Barnett et al. 2001; Capela et al. 

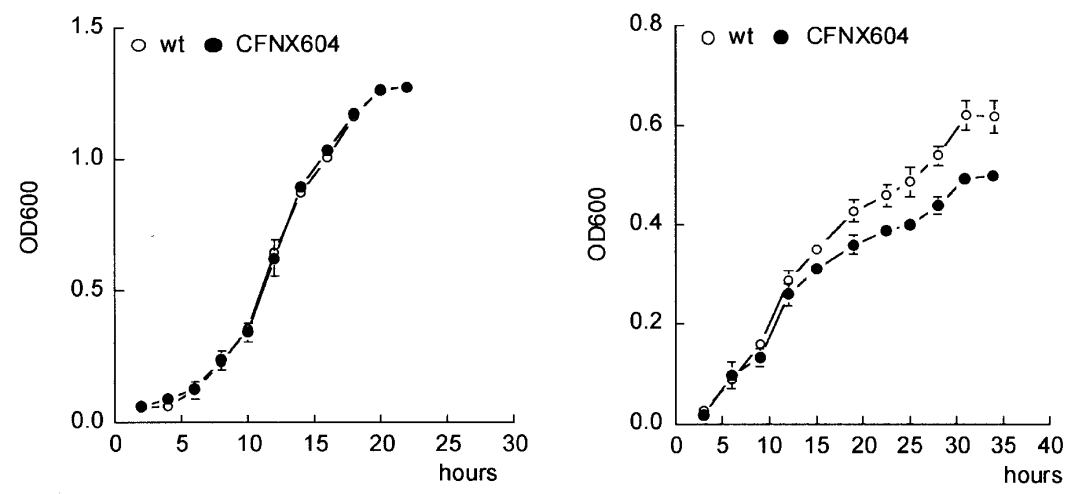

$\mathbf{A}$

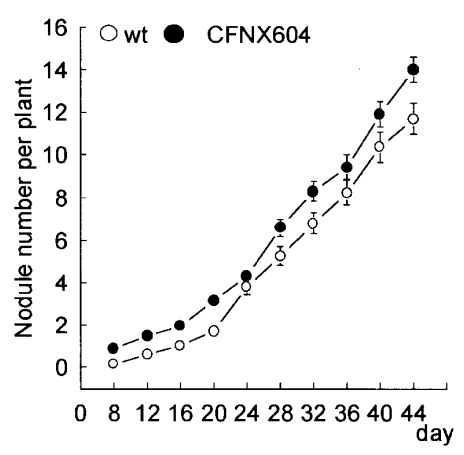

C
B

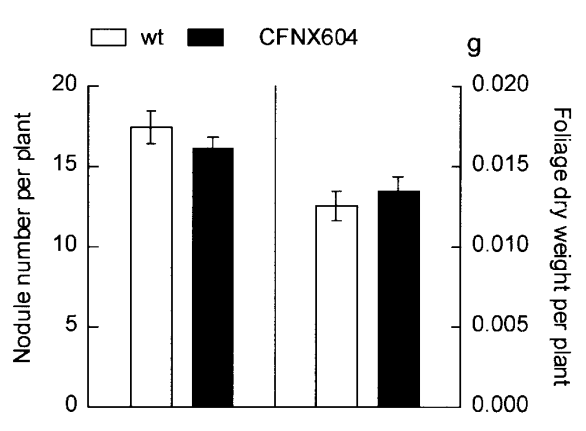

D

Figure 5 Growth and nodulation capacity of wild-type S. meliloti and a derivative strain presenting a three-replicon cointegration (CFNX604). (A) Kinetics of growth in PY medium, (B) kinetics of growth in minimal medium, $(C)$ kinetics of nodulation, and $(D)$ nodules formed and foliage dry weight in Medicago truncatula cv. Jemalong inoculated with S. meliloti 1021 and derivative strain.

2001; Finan et al. 2001; Galibert et al. 2001) allowed us to apply this strategy to the complete genome of $S$. meliloti. Since this approach is based on a general biological process, homologous recombination, it might be applied to any type of microbial genome.

More than 100 bacteria have been sequenced to date, and only a subset of them might be amenable to classical genetic approaches. Conceivably the strategy described here might be used to isolate defined rearrangements of a genome of interest. In particular, searching for derivatives harboring deletions and amplifications might prove useful for fast-screening functional genomics.

\section{METHODS}

\section{Culture Conditions}

Unless otherwise indicated, S. meliloti 1021 and its derivatives were cultivated in PY medium $(0.5 \%$ peptone, $0.3 \%$ yeast extract, and $10 \mathrm{mM} \mathrm{CaCl}_{2}$ ) at $28^{\circ} \mathrm{C}$.

\section{DNA Manipulations, Plasmid Profiles, and PFGE}

Total DNA was prepared using GenomicPrep Cells and a Tissue DNA Isolation Kit (Amersham Biosciences). Plasmid profiles were performed as described by Hynes and McGregor (1990). Agarose gel electrophoresis and filter blot hybridization were carried out as reported (Flores et al. 1987). Radioprobes were labeled with $\mathrm{P}^{32}$ using a rediprime kit (Amersham Pharmacia). The process of mak- the element. The position at the 5 -end of the primer according to the annotation (http://sequence. toulouse.inra.fr/meliloti.html) is shown in parentheses (see Supplemental Material). The sequences of primers for assaying the relative concentration of rearrangements in the experiments presented in Figure 4 and Table 1 are as follows (the name of the primer is indicated in parentheses): primer $1,5^{\prime}$-CATCGGC TTTATCGTACTCGG-3' (ASma3014[3'][1020188]); primer 2, 5' - GCGAACCACTCCTTTCCTAAG-3' (ASma3014 [5'][1025396]); primer 3, 5'-TCCGAGGCTTGCTCACTAAGG-3' (AnodPQ[5'][469884]); primer 4, 5'-TATCTCACGCTTGGAAACA GC-3' (AnodPQ[3'][477039]); primer 5, 5'-ACCCGACCGTTT CCTATTATC-3' (BSmb21705[3'][1571521]); primer 6, 5'-CAGC AGATGTTGTCTACCGCC-3' (BSmb21705[5'][1577411]); primer 7, 5'-ATCTCGGTTACGGTCTTGGAG-3' (BnodPQ[5'][785876]); primer 8, 5'-GCGGATCTCGTCTCTGAACTC-3' (BnodPQ[3'][790533]).

\section{Relative Concentration of Rearrangements}

To quantify the relative proportion of cells presenting a particular rearrangement in a bacterial culture, a PCR-based methodology was used. A culture pure for the specific rearrangement is used as control. As explained in the text, the pure cultures contain cells that have reverted to the original structure; however, this is negligible, since reversions are usually found in less than $10^{-2}$ cells. Total DNA from the control and the test culture is used as templates for the PCR reaction. The PCR reaction is performed for different cycles. When the PCR reaction is in exponential stage, the relative proportion of rearranged structures (F)

\section{Genome Research}


can be obtained by the formula $\mathrm{F}=2^{-\mathrm{n}}$ where $\mathrm{n}$ is the difference in the number of cycles necessary to produce the same amount of PCR product from the control and the test DNA templates.

\section{Bacterial Growth Kinetics and Plant Experiments}

Bacterial strains were propagated in 250-mL flasks containing 50 mL of minimal RMM3 (Price et al. 1992) or PY media inoculated to an initial optical density (OD) at $600 \mathrm{~nm}$ of 0.02 .

For the plant experiments, seeds from Medicago truncatula $\mathrm{cv}$. Jemalong were surface-sterilized and germinated on wet filter papers in petri dishes. After $4 \mathrm{~d}$, plantlets were transferred to plastic growth pouches (Seed-Pack Growth Pouch; Vaughan's Seed) or onto 20 -cm-dia plastic pots containing sterile vermiculite. Each seedling was inoculated with $1 \mathrm{~mL}$ of bacterial suspension. The bacterial inoculum was prepared from a washed overnight culture grown on rich medium and adjusted to 1.0 OD 540 $\mathrm{nm}$. Plants were incubated at $21 \pm 2^{\circ} \mathrm{C}$ with a 16 -h photoperiod provided by cool white fluorescent lights $(110.4 \mu \mathrm{mol}$. $\mathrm{m}-2 \cdot \mathrm{s}-1$ ) and were watered with Jensen liquid medium as necessary. The nodulation kinetics were analyzed using 50 plants per strain. Nodules were counted every $4 \mathrm{~d}$. The total number of nodules and the foliage dry weight of plants grown in pots were analyzed $44 \mathrm{~d}$ after inoculation using 50 plants per strain.

\section{ACKNOWLEDGMENTS}

We thank Angeles Moreno, Marisa Rodríguez, Rosa M. Ocampo, and Virginia Quinto for technical assistance. This work was supported in part by grant ER025/028 from CONACyT-México.

The publication costs of this article were defrayed in part by payment of page charges. This article must therefore be hereby marked "advertisement" in accordance with 18 USC section 1734 solely to indicate this fact.

\section{REFERENCES}

Anderson, R.P. and Roth, J.R. 1977. Tandem genetic duplications in phage and bacteria. Annu. Rev. Microbiol. 31: 473-504.

Barnett, M., Fisher, R.F., Jones, T., Komp, C., Pia Abola, A., Barloy-Hubler, F., Bowser, L., Capela, D., Galibert, F., Gouzy, J., et al. 2001. Nucleotide sequence and predicted functions of the entire Sinorhizobium meliloti pSymA megaplasmid. Proc. Natl. Acad. Sci. 98: 9883-9888.

Capela, D., Barloy-Hubler, F., Gouzy, J., Bothe, G., Ampe, F., Batut, J., Boistard, P., Becker, A., Boutry, M., Cadieu, E., et al. 2001. Analysis of the chromosome sequence of the legume symbiont Sinorhizobium meliloti strain 1021. Proc. Natl. Acad. Sci. 98: 9877-9882.

Finan, T.M., Weider, S., Wong, K., Buhrmester, J., Chain, P., Vorhölter, F.J., Hernández-Lucas, I., Becker, A., Cowie, A., Gouzy, J., et al. 2001.
The complete sequence of the 1,683-kb pSymB megaplasmid from the $\mathrm{N}_{2}$-fixing endosymbiont Sinorhizobium meliloti. Proc. Natl. Acad. Sci. 98: 9889-9894.

Flores, M., González, V., Brom, S., Martínez, E., Piñero, D., Romero, D., Dávila, G., and Palacios, R. 1987. Reiterated DNA sequences in Rhizobium and Agrobacterium spp. J. Bacteriol. 169: 5782-5788.

Flores, M., González, V., Pardo, M.A., Leija, A., Martínez, E., Romero, D. Piñero, D., Dávila, G., and Palacios, R. 1988. Genomic instability in Rhizobium phaseoli. J. Bacteriol. 170: 1191-1196.

Flores, M., Mavingui, P., Perret, X., Broughton, W.J., Romero, D. Hernández, G., Dávila, G., and Palacios, R. 2000. Prediction, identification, and artificial selection of DNA rearrangements: Toward a natural genomic design. Proc. Natl. Acad. Sci. 97: 91389143.

Fraser, C.M., Eisen, J.A., Nelson, K.E., Paulsen, I.T., and Salzberg, S.L. 2002. The value of complete microbial genome sequence. J. Bacteriol. 184: 6403-6405.

Freiberg, C., Fellay, R., Bairoch, A., Broughton, W.J., Rosenthal, A., and Perret, X. 1997. Molecular basis of symbiosis between Rhizobium and legumes. Nature 387: 394-401.

Galibert, F., Finan, T.M., Long, S.R., Pühler, A., Abola, P., Ampe, F., Barloy-Hubler, F., Barnett, M.J., Becker, A., Boistard, P., et al. 2001. The composite genome of the legume symbiont Sinorhizobium meliloti. Science 293: 668-672.

Hynes, M.F. and McGregor, N.F. 1990. Two plasmids other than the nodulation plasmid are necessary for the formation of nitrogen-fixing nodules in Rhizobium leguminosarum. Mol. Microbiol. 4: $567-574$.

Kaneko, T., Nakmura, Y., Sato, S., Asamizu, E., Kato, T., Sasamoto, S., Watanabe, A., Idesawa, K., Ishikawa, A., Kawashima, K., et al. 2000. Complete genome structure of the nitrogen-fixing symbiotic bacterium Mesorhizobium loti. DNA Res. 7: 331-338.

Mavingui, P., Flores, M., Guo, X., Dávila, G., Perret, X., Broughton, W.J., and Palacios, R. 2002. Dynamics of genome architecture in Rhizobium sp. strain NGR234. J. Bacteriol. 184: 171-176.

Petes, T.D. and Hill, C.W. 1988. Recombination between repeated genes in microorganisms. Annu. Rev. Genet. 22: 147-168.

Price, N.P.J., Relick, B., Talmont, F., Lewin, A., Promé, D., Pueppke, S.G., Maillet, F., Dénarié, J., Promé J.-C., and Broughton, W.J. 1992. Broad-host-range Rhizobium species strain NGR234 secrets a family of carbamoylated and fucosylated, nodulation signals that are O-acetylated or sulphated. Mol. Microbiol. 6: 3575-3584.

Romero, D. and Palacios, R. 1997. Gene amplification and genomic plasticity in prokaryotes. Annu. Rev. Genet. 31: 91-111.

\section{WEB SITE REFERENCES}

http://sequence.toulouse.inra.fr/meliloti.html; for the nucleotide sequence of the $S$. meliloti 1021 genome.

Received February 12, 2003; accepted in revised form June 4, 2003. 


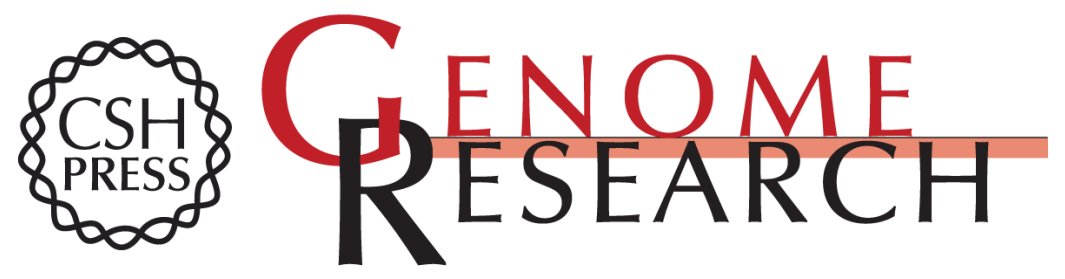

\section{Natural Genomic Design in Sinorhizobium meliloti: Novel Genomic Architectures}

Xianwu Guo, Margarita Flores, Patrick Mavingui, et al.

Genome Res. 2003 13: 1810-1817

Access the most recent version at doi:10.1101/gr.1260903

Supplemental Material

References

License

Email Alerting Service
http://genome.cshlp.org/content/suppl/2003/07/09/13.8.1810.DC1

This article cites 16 articles, 9 of which can be accessed free at: http://genome.cshlp.org/content/13/8/1810.full.html\#ref-list-1

Receive free email alerts when new articles cite this article - sign up in the box at the top right corner of the article or click here.

\section{Affordable, Accurate Sequencing.}

To subscribe to Genome Research go to: https://genome.cshlp.org/subscriptions 